\title{
CONTEXT-FREE WORD PROBLEM SEMIGROUPS
}

\author{
TARA BROUGH, ALAN J. CAIN, AND MARKUS PFEIFFER
}

\begin{abstract}
This paper studies the classes of semigoups and monoids with context-free and deterministic context-free word problem. First, some examples are exhibited to clarify the relationship between these classes and their connection with the notions of word-hyperbolicity and automaticity. Second, a study is made of whether these classes are closed under applying certain semigroup constructions, including direct products and free products, or under regressing from the results of such constructions to the original semigroup(s) or monoid(s).
\end{abstract}

\section{INTRODUCTION}

The deep connections between formal language theory and group theory are perhaps most clearly evidenced by the famous 1985 theorem of Muller and Schupp, which says that a group has context-free word problem if and only if it is virtually free [MS83, Dun85]; indeed, virtually free groups have deterministic context-free word problem. Since then, many studies have analyzed the classes of groups with word problems in various families of formal languages. Herbst \& Thomas characterized the groups with onecounter word problem [HT93, Theorem 5.1]. (For a later elementary proof of this result, see [HOT08].) The first author of the present paper investigated groups whose word problem is an intersection of finitely many context-free languages [Bro14, Bro10]. Holt et al. studied the class of groups whose coword problem is context-free [HRRT99] and Holt and Röver studied the the class of groups whose co-word problem is indexed HR06.

The word problem of a group is the language of words representing the identity over some set of generators and their inverses. Thus two words $u$ and $v$ are equal in a group $G$ if and only if $u V$ is in the word problem, where $V$ is obtained from $v$ by replacing each symbol by its inverse and reversing the word. A natural question is how to generalize this definition to semigroups. Duncan and Gilman [DG99, Definition 5.1] defined the word

The first author was supported by the FCT (Fundação para a Ciência e a Tecnologia/Portuguese Foundation for Science and Technology) fellowship SFRH/ BPD/121469/2016 and by the FCT project UID/Multi/04621/2013.

The second author was supported by an Investigador FCT fellowship (IF/01622/2013/CP1161/CT0001).

For the first and second authors, this work was partially supported by FCT projects UID/MAT/00297/2019 (Centro de Matemática e Aplicações), PTDC/MHCFIL/2583/2014 and PTDC/MAT-PUR/31174/2017.

This work was started during a visit by the third author to the Universidade Nova de Lisboa, which was supported by the exploratory project IF/01622/2013/CP1161/CT0001 attached to the second author's research fellowship. 
problem of a semigroup $S$ with respect to a generating set $A$ to be

$$
\mathrm{WP}(S, A)=\left\{u \# v^{\mathrm{rev}}: u, v \in A^{+}, u={ }_{S} v\right\},
$$

where $v^{\text {rev }}$ is the reverse of $v$. This definition fits well with the group definition and is natural when considering word problems recognizable by automata equipped with a stack. It was used by Holt, Owens, and Thomas in their study of groups and semigroups with one-counter word problem HOT08, and by Hoffmann et al. in their study of semigroups with contextfree word problem [HHOT12].

The main conclusions of Hoffmann et al.'s earlier study were the result that the class of semigroups with context-free word problem is closed under passing to finite Rees index subsemigroups and extensions HHOT12, Theorem 1] and a characterization of completely simple semigroups with context-free word problem as Rees matrix semigroups over virtually free groups [HHOT12, Theorem 2].

This paper explores new directions in the study of the class of semigroups with context-free word problem, including monoids with context-free word problem, and also considers the classes of semigroups and monoids with deterministic context-free word problem. First, Section 3 exhibits some natural classes of semigroups and monoids that lie within and outside these classes; in particular Example 3.3 shows that having context-free and deterministic context-free word problem do not coincide for semigroups or monoids, unlike (as noted above) for groups. Section 4 discusses connections with the theories of word-hyperbolic and automatic semigroups: any semigroup or monoid with context-free word problem is word-hyperbolic, but there are non-automatic semigroups that have context-free word problem. The remainder of the core of the paper (Sections 5 8) focusses on various constructions: direct products, free products, strong semilattices of semigroups, Rees matrix semigroups and Bruck-Reilly extensions. For each construction, the questions of interest are: (1) Are the classes of semigroups and monoids with context-free or deterministic context-free word problem closed under that construction? (2) If the result of applying such a construction lies in one of these classes, must the original semigroup(s) or monoids(s) lie in that same class? Finally, Section 10 lists some open problems.

\section{Preliminaries}

The word problem for a semigroup $S$ is defined as (1.1) above. Similarly, the word problem for a monoid $M$ with respect to a generating set $A$ is the language

$$
\mathrm{WP}(M, A)=\left\{u \# v^{\mathrm{rev}}: u, v \in A^{*}, u={ }_{M} v\right\} .
$$

Proposition 2.1 ([HHOT12, Proposition 8]). Let $\mathfrak{C}$ be a class of languages closed under inverse homomorphisms and intersection with regular languages. Then

(1) If a semigroup or monoid has word problem in $\mathfrak{C}$ with respect to some generating set, then it has word problem in $\mathfrak{C}$ with respect to any generating set. 
(2) The class of semigroups (resp. monoids) with word problem in $\mathfrak{C}$ is closed under taking finitely generated subsemigroups (resp. submonoids).

The preceding result applies in particular when $\mathfrak{C}$ is the class of contextfree or deterministic context-free languages [GG66, HU79].

If a semigroup (resp. monoid) has word problem in a class of languages $\mathfrak{C}$, it is said to be a $U(\mathfrak{C})$ semigroup (resp. monoid). We denote the classes of context-free and deterministic context-free languages by $\mathcal{C F}$ and $\mathcal{D C F}$ respectively. The ' $U$ ' notation is because (1.1) and (2.1) treat the word problem as an 'unfolded' relation rather than a 'two-tape' relation; see [BC18] for a systematic study.

\section{EXAMPLES}

We recall some less commonly-used terms from the theory of rewriting systems; see [BO93] for general background. A rewriting system $(A, \mathcal{R})$ is monadic if it is length-reducing and the right-hand side of each rewrite rule in $\mathcal{R}$ lies in $A \cup\{\varepsilon\}$. A monadic rewriting system $(A, \mathcal{R})$ is regular (respectively, context-free) if, for each $a \in A \cup\{\varepsilon\}$, the set of all left-hand sides of rewrite rules in $\mathcal{R}$ with right-hand side $a$ is a regular (respectively, context-free) language.

Theorem 3.1 ([CM12, Theorem 3.1]). Let $(A, \mathcal{R})$ be a confluent context-free monadic rewriting system. Then the monoid presented by $\langle A \mid \mathcal{R}\rangle$ is $U(\mathcal{C F})$, and a context-free grammar generating its word problem can be effectively constructed from context-free grammars describing $\mathcal{R}$.

(The preceding result originally stated that a monoid satisfying the hypothesis was word-hyperbolic; however, the proof proceeds by constructing the word problem for the monoid. The 'effective construction' part follows easily by inspecting the construction in the proof.)

Example 3.2. This example shows that a $U(\mathcal{C F})$ monoid need not have a context-free cross section (that is, a language over some generating set containing a unique representative for every element).

Let $K=\left\{a^{\alpha} b^{\alpha} c^{\alpha}: \alpha \in \mathbb{N} \cup\{0\}\right\}$ and let $L=\{a, b, c\}^{*}-K$. It is wellknown that $K$ is not a context-free language but that $L$ is a context-free language. Let $A=\{a, b, c, x, y, z\}$ and let $\mathcal{R}=\{(x w y, z): w \in L\}$. Let $M$ be the monoid presented by $\langle A \mid \mathcal{R}\rangle$. By Theorem 3.1, $M$ is $U(\mathcal{C F})$. Suppose that $M$ admits a context-free cross-section. Then $M$ admits a context-free cross-section $J \subseteq A^{*}$. Let $u$ be the unique word in $J$ such that $u={ }_{M} z$, and let $J^{\prime}=(J \backslash\{u\}) \cup\{z\}$; then $J^{\prime}$ is also a context-free cross-section of $M$. Let $H=J^{\prime} \cap x\{a, b, c\}^{*} y$. Then $H$ is context-free and comprises precisely the words $x w y$ where $w \in K$, for if $w \in L$, then $x w y={ }_{M} z$, and the representative of $z$ in $J^{\prime}$ is the word $z$ itself. Hence, since the class of context-free languages is closed under right and left quotients with regular sets, $K=x \backslash H / y$ is context-free. This is a contradiction, and so $M$ does not admit a context-free cross-section.

Example 3.3. This example shows that the class of $U(\mathcal{D C F})$ semigroups is properly contained in the class of $U(\mathcal{C F})$ semigroups. 
Let $K$ be the language of palindromes over $\{a, b\}$. It is well-known that $K$ is context-free but not deterministic context-free. Let $A=\{a, b, x, y, z\}$ and let $\mathcal{R}=\{(x w y, z): w \in L\}$. Let $M$ be the monoid presented by $\langle A \mid \mathcal{R}\rangle$.

By Theorem 3.1, $M$ is $U(\mathcal{C F})$. Suppose, with the aim of obtaining a contradiction, that $M$ is $U(\mathcal{D C F})$. Then $\mathrm{WP}(M, A)$ is deterministic contextfree. Let $L=\left(\operatorname{WP}(M, A) \cap A^{*} \# z\right) /\{\# z\} \cap\{a, b, x, y\}^{*}$; then $L$ is the language of words over $\{a, b, x, y\}$ that are equal to $z$ in $M$. Furthermore, $L$ is deterministic context-free, since the class of deterministic context-free languages is closed under intersection with regular languages [HU79, Theorem 10.4] and right quotient by regular languages [HU79, Theorem 10.2].

Now, $K=x \backslash L / y$. The class of determinstic context-free languages is closed under left quotient by a singleton (since a deterministic pushdown automaton can simulate reading a fixed word before it starts reading input), and, as noted above, is closed under right quotient by regular languages. Hence $K$ is deterministic context-free. This is a contradiction, and so $M$ is not $U(\mathcal{D C F})$.

We conjecture that the bicyclic monoid $B=\langle b, c \mid b c=\varepsilon\rangle$, which is $U(\mathcal{C F})$ by Theorem 3.1, is not $U(\mathcal{D C F})$. To motivate this conjecture, consider the intersection of $\operatorname{WP}(B,\{b, c\})$ with the regular language $c^{*} \# c^{*} b^{*} c^{*}$. Then a deterministic pushdown automaton recognizing this intersection, when reading $c^{\alpha} \# c^{\alpha} b^{\beta} c^{\gamma}$ would have to enter an accept state after reading $c^{\alpha} \# c^{\alpha}$ but could then accept the whole input if and only if $\alpha \geq \beta=\gamma$, and checking two independent comparisons is heuristically impossible for a pushdown automaton.

Example 3.4. An example of a monoid that is 'close' to being a free group but is not $U(\mathcal{C F})$ is the free inverse monoid of rank 1 and hence (by Proposition 2.1) of any finite rank. This follows from applying the pumping lemma to the intersection of the word problem and the regular language $x^{*}\left(x^{-1}\right)^{*} x^{*} \# x^{*}$ (where $x$ is the free generator); see [Bro18, Theorem 1].

\section{RELATIONSHIP TO WORD-HYPERBOLICITY AND AUTOMATICITY}

Hyperbolic groups have become one of the most fruitful areas of group theory since their introduction by Gromov [Gro87]. The concept of hyperbolicity can be generalized to semigroups and monoids in more than one way, but here we consider the linguistic definition that uses Gilman's characterization of hyperbolic groups using context-free languages [Gil02]. A word-hyperbolic structure for a semigroup $S$ is a pair $(L, M(L))$, where $L$ is a regular language over an alphabet $A$ representing a finite generating set for $S$ such that $L$ maps onto $S$, and where

$$
M(L)=\left\{u \#_{1} v \#_{2} w^{\mathrm{rev}}: u, v, w \in L \wedge u v=_{S} w\right\}
$$

(where $\#_{1}$ and $\#_{2}$ are new symbols not in $A$ ) is context-free.

Theorem 4.1. Every $U(\mathcal{C F})$ semigroup is word-hyperbolic.

The proof is in effect the first paragraph of the proof of Theorem 3.1 as given in CM12, Proof of Theorem 2]. We give it here for completeness. 
Proof. Let $S$ be a $U(\mathcal{C F})$ semigroup, and let $A$ be a finite generating set for $S$. Let $\phi:\left(A \cup\left\{\#_{1}, \#_{2}\right\}\right)^{*} \rightarrow\left(A \cup\left\{\#_{1}\right\}\right)^{*}$ be the homomorphism extending $\#_{1} \mapsto \varepsilon, \quad \#_{2} \mapsto \#, \quad a \mapsto a$ for all $a \in A$.

Then $M\left(A^{*}\right)=(\mathrm{WP}(S, A)) \phi^{-1} \cap\left(A^{*} \#_{1} A^{*} \#_{2} A^{*}\right)$. Since the class of contextfree languages is closed under taking inverse homomorphisms, $M\left(A^{*}\right)$ is also context-free. Hence $\left(A^{*}, M\left(A^{*}\right)\right)$ is a word-hyperbolic structure for $S$.

All hyperbolic groups are automatic $\left[\mathrm{ECH}^{+} 92\right.$, Theorem 3.4.5], but wordhyperbolic semigroups may not even be asynchronously automatic HKOT02, Example 7.7]. Even within the smaller class of $U(\mathcal{C F})$ semigroups, one can find semigroups that are not automatic:

Example 4.2. Let $A=\{a, b, c, d, z\}$, let $\mathcal{R}=\left\{\left(a b^{\alpha} c^{\alpha} d, z\right): \alpha \in \mathbb{N}\right\}$. Let $M$ be the monoid presented by $\langle A \mid \mathcal{R}\rangle$. Then $M$ is $U(\mathcal{C F})$ by Theorem 3.1. but cannot be automatic [CRRT01, Corollary 5.5]. (In fact, it can be shown that $M$ is not even asynchronously automatic.)

Given that $U(\mathcal{C F})$ groups are virtually free and thus automatic, and since the monoid in Example 4.2 is not cancellative, the following question is natural:

Question 4.3. Is a cancellative $U(\mathcal{C F})$ semigroup necessarily automatic?

\section{Direct products}

A direct product of two finitely generated semigroups is not necessarily finitely generated. However, a direct product of two $U(\mathcal{C F})$ semigroups is not necessarily $U(\mathcal{C F})$, even if it is finitely generated: for example, the free monoid of rank 1 is $U(\mathcal{C F})$, but the direct product of two copies of this monoid is the free commutative monoid of rank 2 , which is finitely generated but not $U(\mathcal{C F})$.

For a semigroup $S$, we say that $S$ is decomposable if $S^{2}=S$. We will show that for a direct product of two $U(\mathcal{C F})$ semigroups to be $U(\mathcal{C F})$, it is necessary and sufficient that one of the factors is finite and decomposable (decomposability being necessary to ensure finite generation). First we establish sufficiency.

Lemma 5.1. The classes of $U(\mathcal{C F})$ and $U(\mathcal{D C F})$ semigroups are closed under taking direct product with a finite decomposable semigroup.

Proof. Let $S$ be a $U(\mathcal{C F})$ semigroup and $T$ a finite decomposable semigroup. Then $S \times T$ is finitely generated ([RRW98, Theorem 8.2]). Let $C$ be a finite generating set for $S \times T$ and let $A$ and $B$ be the projections of $C$ onto $S$ and $T$ respectively. Then $A$ and $B$ are finite generating sets for $S$ and $T$ respectively. Thus there exists a pushdown automaton $\mathcal{A}$ recognising $\mathrm{WP}(S, A)$, which can be modified to give a pushdown automaton $\mathcal{A}^{\prime}$ recognising $\mathrm{WP}(S \times T, A \times B)$, by processing the symbols from $A$ as usual, while using the states to record the finite information required to check validity of the input on the second tape. Hence $S \times T$ is $U(\mathcal{C F})$. Moreover, if $S$ is $U(\mathcal{D C F})$, then $\mathcal{A}$ can be taken to be deterministic, in which case $\mathcal{A}^{\prime}$ is also deterministc, so $S \times T$ is $U(\mathcal{D C F})$. 
Necessity arises from the following language-theoretic result, which encapsulates the idea that context-free languages cannot admit 'cross-dependencies'. For words $w, w^{\prime}$, we use the notation $w^{\prime} \sqsubseteq w$ to mean that $w^{\prime}$ is a subword of $w$.

Lemma 5.2. Let $A$ and $B$ be disjoint alphabets, and let $\rho_{A}, \rho_{B}$ be equivalence relations on $A^{*}$ and $B^{*}$ respectively with infinitely many equivalence classes. Then the language $L\left(\rho_{A}, \rho_{B}\right)=\left\{u_{1} v_{1} u_{2} v_{2}:\left(u_{1}, u_{2}\right) \in \rho_{A},\left(v_{1}, v_{2}\right) \in\right.$ $\left.\rho_{B}\right\}$ is not context-free.

Proof. Suppose that $L=L\left(\rho_{A}, \rho_{B}\right)$ is context-free, and let $k$ be the pumping constant for $L$. Let $\mathcal{E}_{A}$ be the set of all equivalence classes of $\rho_{A}$ that contain a word of length at most $k$, and define $\mathcal{E}_{B}$ similarly.

Let $w=u_{1} v_{1} u_{2} v_{2} \in L$ with $\left|v_{1}\right|,\left|u_{2}\right|>k$. Then we can write $w=p q r s t$ where $|q r s| \leq k,|q s| \geq 1$ and $p q^{i} r s^{i} t \in L$ for all $i \in \mathcal{N}_{0}$. Due to the form of words in $L, q$ and $s$ must each be a subword of some $u_{i}$ or $v_{i}$. Moreover, the lengths of $u_{2}$ and $v_{1}$ preclude the possibility that $q \sqsubseteq u_{1}$ and $s \sqsubseteq u_{2}$ or $p \sqsubseteq v_{1}$ and $q \sqsubseteq v_{2}$. Let $w^{\prime}=p r t=u_{1}^{\prime} v_{1}^{\prime} u_{2}^{\prime} v_{2}^{\prime}$. Then we have $u_{i}^{\prime}=u_{i}$ for some $i \in\{1,2\}$ and $v_{j}^{\prime}=v_{j}$ for some $j \in\{1,2\}$. Since $w^{\prime} \in L$, this implies that the equivalence classes of the factors are unchanged between $w$ and $w^{\prime}$. By induction, we can repeat this process until we obtain a word $w^{b}=u_{1}^{b} v_{1}^{b} u_{2}^{b} v_{2}^{b} \in L$ with $\left|v_{1}^{b}\right| \leq k$ or $\left|u_{2}^{b}\right| \leq k$, where the $u_{i}^{b}$ are in the same $\rho_{A}$-equivalence class as the $u_{i}$ and the $v_{i}^{b}$ are in the same $\rho_{B}$-equivalence class as the $v_{i}$. Hence our original word $w$ had either $u_{i} \in C$ for some $C \in \mathcal{E}_{A}$ or $v_{i} \in D$ for some $D \in \mathcal{E}_{B}$. But $\mathcal{E}_{A}$ and $\mathcal{E}_{B}$ are both finite, and so $L$ cannot contain all words of the form $u_{1} v_{1} u_{2} v_{2}$ with $\left(u_{1}, u_{2}\right) \in \rho_{A}$ and $\left(v_{1}, v_{2}\right) \in \rho_{B}$. Hence $L$ is not context-free.

The preceding lemma is immediately applicable only to monoids.

Lemma 5.3. The direct product of two infinite monoids cannot be $U(\mathcal{C F})$.

Proof. Let $S=\langle A\rangle$ and $T=\langle B\rangle$ be infinite monoids. Then the relations $\rho_{A}=\iota(S, A)$ and $\rho_{B}=\iota(T, B)$ both have infinitely many equivalence classes. Moreover, the language $L=\mathrm{WP}(S \times T, A \cup B) \cap A^{*} B^{*} \# A^{*} B^{*}$ has as a homomorphic image the language $L\left(\rho_{A}, \rho_{B}\right)$ defined in Lemma 5.2. Since the class of context-free languages is closed under homorphisms and intersection with regular sets, this implies that $S \times T$ is not $U(\mathcal{C F})$.

Thus if $S \times T$ is $U(\mathcal{C F})$, then at least one of $S$ or $T$ is finite.

In order to extend Lemma 5.3 to all semigroups, we first establish the following fact (which is clear for monoids, where direct factors are submonoids).

Lemma 5.4. The class of $U(\mathcal{C F})$ semigroups is closed under taking direct factors.

Proof. Assume that $S \times T$ is $U(\mathcal{C F})$. In particular, $S \times T$ is finitely generated. By [RRW98, Theorem 2.1], $S$ and $T$ are finitely generated, and $S^{2}=S$ and $T^{2}=T$.

Let $C=\left\{c_{1}, \ldots, c_{k}\right\}$ be a finite generating set for $T$. Since $T^{2}=T$, we can choose a factorization $c_{i}=c_{i \zeta} u_{i}$ for each $c_{i} \in C$. Construct a labelled digraph with vertex set $C$ and an edge from $c_{i}$ to $c_{i \zeta}$ labelled by $u_{i}$ for each $c_{i} \in C$. Since this digraph is finite, it must contain a circuit. Fix some 
vertex $c$ on that circuit and let $w$ be the concatenation in reverse order of the labels on the edges around the circuit. Then $c w=c$.

Let $A$ be a finite generating set for $S \times T$ and let $B$ be a finite generating set for $S$. Then $X=A \cup(B \times\{c, w\})$ is a finite generating set for $S \times T$. Let $R$ be the regular language $(B \times\{c\})(B \times\{w\})^{*} \#(B \times\{w\})^{*}(B \times\{c\})$. Let $L=\mathrm{WP}(S \times Y, X) \cap R$. Then

$$
\begin{aligned}
& \left(b_{1}, c\right)\left(b_{2}, w\right) \cdots\left(b_{m}, w\right) \#\left(b_{n}^{\prime}, w\right) \cdots\left(b_{2}^{\prime}, w\right)\left(b_{1}, c\right) \in L \\
\Longleftrightarrow & \left(b_{1} b_{2} \cdots b_{m}, c w^{m-1}\right)={ }_{S \times T}\left(b_{1}^{\prime} b_{2}^{\prime} \cdots b_{n}^{\prime}, c w^{n-1}\right) \\
\Longleftrightarrow & \left(b_{1} b_{2} \cdots b_{m}, c\right)={ }_{S \times T}\left(b_{1}^{\prime} b_{2}^{\prime} \cdots b_{n}^{\prime}, c\right) \\
\Longleftrightarrow & b_{1} b_{2} \cdots b_{m}={ }_{S} b_{1}^{\prime} b_{2}^{\prime} \cdots b_{n}^{\prime} .
\end{aligned}
$$

Define a homomorphism

$$
\pi:((B \times\{c, w\}) \cup\{\#\}) \rightarrow(B \cup\{\#\}), \quad(b,\lrcorner) \mapsto b, \quad \# \mapsto \# .
$$

Then (5.1) shows that $L \pi=\operatorname{WP}(S, B)$. Since the class of context-free languages is closed under homomorphism [HU79, Corollary to Theorem 6.2], $S$ is a $U(\mathcal{C F})$ semigroup.

Theorem 5.5. The direct product of two semigroups is $U(\mathcal{C F})$ if and only if it is finite or one of the factors is $U(\mathcal{C F})$ and the other factor is finite and decomposable.

Proof. Sufficiency was already established in Lemma 5.1

Conversely suppose that $S \times T$ is $U(\mathcal{C F})$. Let $C$ be a finite generating set for $S \times T$ with the projection of $C$ onto the first component being $A$ and the projection onto the second component $B$. By Lemma 5.4, $S$ and $T$ are both $U(\mathcal{C F})$. Let $A_{1}=A \times\{1\}, B_{1}=\{1\} \times B$, and $C_{1}=A_{1} \cup B_{1} \cup C$. We will describe a pushdown automaton $\mathcal{P}$ recognising $\mathrm{WP}\left(S^{1} \times T^{1}, C_{1}\right)$. This automaton is defined in terms of pushdown automata $\mathcal{A}, \mathcal{B}$ and $\mathcal{C}$, recognising $\mathrm{WP}(S, A), \mathrm{WP}(T, B)$ and $\mathrm{WP}(S \times T, C)$ respectively.

On input $(x, y) \in C$, the automaton $\mathcal{P}$ behaves as a 'delayed' version of $\mathcal{C}$, storing the input symbol in the state and then (except in the start state, which has no stored symbol) simulating $\mathcal{C}$ on input of the current stored symbol. The automaton may guess at any point that the input is complete, and process the stored symbol from the current state as an $\epsilon$-transition. In this case we move to a state with no stored symbol and accepting no further input, which is a final state if and only if it is a final state in $\mathcal{C}$. Thus on input in $(C \cup\{\#\})^{*}, \mathcal{P}$ behaves exactly like $\mathcal{C}$ but 'one step behind', and so the sublanguage of $(C \cup\{\#\})^{*}$ accepted by $\mathcal{P}$ is $\operatorname{WP}(S \times T, C)$.

In order to work with input from $A_{1} \cup B_{1}$ we choose, for all $x, x^{\prime} \in A$ and $y, y^{\prime} \in B$, representatives $w_{x, x^{\prime}, y}$ and $w_{x, y, y^{\prime}}$ in $C$ for the elements $\left(x x^{\prime}, y\right)$ and $\left(x, y y^{\prime}\right)$ of $S \times T$.

Now, if the automaton $\mathcal{P}$ reads the symbol $\left(x^{\prime}, 1\right)$ in a state with stored symbol $(x, y)$, it simulates reading all but the final symbol of $w_{x, x^{\prime}, y^{\prime}}$ in $\mathcal{C}$ from the current state, and stores the final symbol in the last state of this computation. Symmetrically, the same occurs when we replace $\left(x^{\prime}, 1\right)$ by $\left(1, y^{\prime}\right)$ and $w_{x, x^{\prime}, y}$ by $w_{x, y, y^{\prime}}$. Thus on input $u \# v$ from $C C_{1}^{*} \# C C_{1}^{*}$, the automaton is able to simulate processing in $\mathcal{C}$ some $u^{\prime} \# v^{\prime}$ such that $u={ }_{S \times T}$ $u^{\prime}$ and $v={ }_{S \times T} v^{\prime}$. 
Finally, on input from $A_{1}$ or $B_{1}$ in the start state, the automaton guesses whether the remaining (non-\#) input will be in $A_{1}^{*}$ resp. $B_{1}^{*}$. If it guesses yes, it moves to a copy of the appropriate automaton $\mathcal{A}$ resp. $\mathcal{B}$, treating input $(x, 1)$ as $x$ and $(1, y)$ as $y$. Thus the sublanguage of $\left(A_{1} \cup\{\#\}\right)^{*}$ recognised by $\mathcal{P}$ is $\operatorname{WP}\left(S \times\{1\}, A_{1}\right)$, while the sublanguage of $\left(B_{1} \cup\{\#\}\right)^{*}$ recognised is $\mathrm{WP}\left(\{1\} \times T, B_{1}\right)$. If, on the other hand, the automaton guesses no, we describe what happens on input from $A_{1}$, the other case being symmetric. Supposing the input is $(x, 1)$, the automaton guesses which $y \in B$ will be read next, and stores this guess in the state, along with the symbol $(x, y)$. States with a stored guess $y \in B$ operate as usual, except on input of the form $(x, y)$. On such input, the automaton deletes the 'guess' $y$ and otherwise operates as if the input were $(x, 1)$, since it already simulated reading $y$ earlier. (If $x=1$, then we simply delete the guess and otherwise do nothing.) The automaton must similarly make a guess on input from $A_{1}$ or $B_{1}$ in a state with stored symbol \#. Since $(x, 1) w\left(x^{\prime}, y\right)=(x, y) w\left(x^{\prime}, 1\right)$ for $w \in A_{1}^{*}$, the automaton $\mathcal{P}$ is now able to simulate reading a corresponding word in $C^{*}$ for any input not in $\left(A_{1} \cup\{\#\}\right)^{*} \cup\left(B_{1} \cup\{\#\}\right)^{*}$. Combined with the fact that $\mathcal{P}$ can also simulate the automata $\mathcal{A}$ and $\mathcal{B}$ on appropriate inputs, this establishes that $\mathcal{P}$ recognises $\operatorname{WP}\left(S^{1} \times T^{1}, C_{1}\right)$.

Thus $S^{1} \times T^{1}$ is $U(\mathcal{C F})$, and so by Lemma 5.3, without loss of generality we can assume $T^{1}$ is finite. Moreover, $S^{1}$ is $U(\mathcal{C F})$, and hence so is $S$, by Proposition 2.1.2. By [RRW98, Theorem 8.1], if $S$ is infinite then $T$ must also be decomposable, since $S \times T$ is finitely generated.

\section{FREE PRODUCTS}

Theorem 6.1. The class of $U(\mathcal{C F})$ semigroups is closed under taking semigroup free products and under taking free factors.

Proof. Let $S$ and $T$ be $U(\mathcal{C F})$ semigroups. Let $A_{S}$ and $A_{T}$ be finite generating sets for $S$ and $T$, respectively, and for $X \in\{S, T\}$, let $\mathcal{P}_{X}$ be a pushdown automaton recognizing $\operatorname{WP}\left(X, A_{X}\right)$ accepting by final state, Assume that in $\mathcal{P}_{X}$, the initial stack content is only a stack bottom symbol $\perp_{X}$, which is never never popped or pushed.

Construct a new pushdown automaton $\mathcal{Q}$ recognizing words over $A_{S} \cup$ $A_{T} \cup\{\#\}$, functioning as follows. First, $\mathcal{Q}$ will recognize words in $\left(A_{S} \cup\right.$ $\left.A_{T}\right)^{+} \#\left(A_{S} \cup A_{T}\right)^{+}$; since this is a regular language, assume without loss that the input is in this form. When $\mathcal{Q}$ begins, it reads a symbol from $A_{X}$ (for some $X \in\{S, T\}$ ). It pushes $\perp_{X}$ onto its stack and begins to simulate $\mathcal{P}_{X}$. Whenever it is simulating $\mathcal{P}_{X}$ and reads a symbol from $A_{Y}$, where $Y \neq X$, it pushes the current state of $\mathcal{P}_{X}$ onto the stack, then pushes $\perp_{Y}$ onto the stack and begins to simulate $\mathcal{P}_{Y}$. These alternating simulations of $\mathcal{P}_{S}$ and $\mathcal{P}_{T}$ continue until the \# is encountered.

On reading the symbol \#, the automaton $\mathcal{Q}$ continues to simulate whichever $\mathcal{P}_{X}$ it was currently simulating. After this point, whenever it is simulating $\mathcal{P}_{X}$ (for some $X \in\{S, T\}$ ) and reads a symbol from $A_{Y}$, where $Y \neq X$, how it proceeds depends on whether the currently-simulated $\mathcal{P}_{X}$ is in an accept state:

- If it is in accept state, $\mathcal{Q}$ pops symbols from its stack until it encounters $\perp_{X}$, which it pops, then pops the state of $\mathcal{P}_{Y}$, restores the 
simulation of $\mathcal{P}_{Y}$ from this state (and with the stack contents down to the symbol $\perp_{Y}$ ), and simulates $\mathcal{P}_{Y}$ on reading \# and then on reading the symbol just read by $\mathcal{Q}$. (If after popping $\perp_{X}$ the stack of $\mathcal{Q}$ is empty, it fails.)

- If it is not in an accept state, $\mathcal{Q}$ fails.

These alternating simulations of $\mathcal{P}_{S}$ and $\mathcal{P}_{T}$ continue until the end of the input unless $\mathcal{Q}$ fails before then. At this point $\mathcal{Q}$ accepts if the currentlysimulated $\mathcal{P}_{X}$ is in an accept state, and if the stack only contains symbols from the stack alphabet $B_{X}$ plus a single symbol $\perp_{X}$.

It follows from the above description that $\mathcal{Q}$ recognizes strings of the form

$$
u_{1} u_{2} \cdots u_{k} \# v_{k}^{\mathrm{rev}} \cdots v_{2}^{\mathrm{rev}} v_{1}^{\mathrm{rev}}
$$

where $u_{i} \# v_{i}^{\text {rev }} \in L\left(\mathcal{P}_{X(i)}\right)$ and either $X(2 j)=S$ and $X(2 j+1)=T$, or else $X(2 j)=T$ and $X(2 j+1)=S$. Thus $\mathcal{Q}$ recognizes strings (6.1) such that

$$
u_{1} u_{2} \cdots u_{k}={ }_{S * T} v_{1} v_{2} \cdots v_{k} \text {, }
$$

and the $u_{i}$ and $v_{i}$ are either both in $A_{X}^{+}$or both in $A_{Y}^{+}$for alternating $i$. Thus $\mathcal{Q}$ recognizes $\mathrm{WP}\left(S * T, A_{X} \cup A_{Y}\right)$.

The free factors of a finitely generated free product are themselves finitely generated, so closure under free factors follows from Proposition [2.1,2.

Notice that the strategy of the proof of Theorem 6.1 cannot be applied to show that the class of $U(\mathcal{D C F})$ semigroups is closed under taking free products. The problem is in the very last step: after the automaton has read its last input symbols from some $A_{X}$, it cannot deterministically check that the stack only contains symbols from the stack alphabet $B_{X}$ plus a single symbol $\perp_{X}$. Therefore the following question remains open:

Question 6.2. Is the class of $U(\mathcal{D C F})$ semigroups closed under forming free products?

Theorem 6.3. The class of $U(\mathcal{C F})$ monoids is closed under taking monoid free products and free factors.

Sketch proof. It is easy to see that the construction of the $\mathcal{Q}$ from the proof of Theorem 6.1 can be adapted to the case of monoid free products. Using the notation from that proof, one observes that for $X \in\{S, T\}$ the language of words over $A_{X}$ representing the identity of $X$ is a context-free language $K_{X}$. Then one first modifies $\mathcal{Q}$ to accept \# (that is, the empty word, followed by \#, followed by the empty word), then modifies $\mathcal{Q}$ so that it can non-deterministically read a string from either $K_{X}$ at any point (including while reading another string from $K_{Y}$ for $Y \in\{S, T\}$, so that such strings can be 'nested').

\section{Strong SEMilattices}

We recall the definition of a strong semilattice of semigroups here, and refer the reader to [How95, Sect. 4.1] for further background reading:

Let $Y$ be a semilattice. Recall that the meet of $\alpha, \beta \in Y$ is denoted $\alpha \wedge \beta$. For each $\alpha \in Y$, let $S_{\alpha}$ be a semigroup. For $\alpha \geq \beta$, let $\phi_{\alpha, \beta}: S_{\alpha} \rightarrow S_{\beta}$ be a homomorphism such that 
(1) For each $\alpha \in Y$, the homomorphism $\phi_{\alpha, \alpha}$ is the identity mapping.

(2) For all $\alpha, \beta, \gamma \in Y$ with $\alpha \geq \beta \geq \gamma, \phi_{\alpha, \beta} \phi_{\beta, \gamma}=\phi_{\alpha, \gamma}$.

The strong semilattice of semigroups $S=\mathcal{S}\left[Y ; S_{\alpha} ; \phi_{\alpha, \beta}\right]$ consists of the disjoint union $\bigcup_{\alpha \in Y} S_{\alpha}$ with the following multiplication: if $x \in S_{\alpha}$ and $y \in S_{\beta}$, then

$$
x y=\left(x \phi_{\alpha, \alpha \wedge \beta}\right)\left(y \phi_{\beta, \alpha \wedge \beta}\right),
$$

where $\alpha \wedge \beta$ denotes the greatest lower bound of $\alpha$ and $\beta$.

Theorem 7.1. Let $\mathfrak{C}$ be a class of languages closed under finite union, inverse gsm-mappings and intersection with regular languages (in particular, the class $\mathcal{C F})$. A strong semilattice of semigroups is $U(\mathfrak{C})$ if and only if it is finitely generated and all the semigroups in its lattice are $U(\mathfrak{C})$.

Proof. Let $S=\mathcal{S}\left[Y ; S_{\alpha} ; \phi_{\alpha, \beta}\right]$ be a strong semilattice of semigroups. If $S$ is $U(\mathfrak{C})$, then it must be finitely generated (that is, $Y$ must be finite and each $S_{\alpha}$ finitely generated). Moreover, the $S_{\alpha}$ must all be $U(\mathfrak{C})$, since they are finitely generated subsemigroups of $S$.

Conversely, suppose that $Y$ is finite and each $S_{\alpha}$ is $U(\mathfrak{C})$. For each $\alpha \in Y$, let $A_{\alpha}$ be a finite generating set for $S_{\alpha}$ and $A_{\alpha}^{\prime}=\bigcup_{\beta>\alpha} A_{\alpha}$. Let $A=$ $\bigcup_{\alpha \in Y} A_{\alpha}$. Define homomorphisms $\phi_{\alpha}:\left(A_{\alpha}^{\prime}\right)^{*} \rightarrow A_{\alpha}^{*}$ by $x \mapsto x \phi_{\beta, \alpha}$ for $x \in A_{\beta}$.

We can view $\operatorname{WP}(S, A)$ as the union of its restrictions to each $S_{\alpha}$ : that is, as the union of the languages $L_{\alpha}=\left\{u \# v^{\mathrm{rev}} \in \mathrm{WP}(S, A): u, v \in S_{\alpha}\right\}$. In turn, each $L_{\alpha}$ can be expressed as $L_{\alpha}^{\prime} \cap R_{\alpha}$, where $L_{\alpha}^{\prime}=\left\{u \# v^{\mathrm{rev}}: u, v \in\right.$ $\left.A^{*}, u \phi_{\alpha}=S_{\alpha} v \phi_{\alpha}\right\}$ and $R_{\alpha}=\left\{u \# v^{\mathrm{rev}}: u, v \in A^{*}, u, v \in S_{\alpha}\right\}$. Note that $u \# v^{\mathrm{rev}} \in L_{\alpha}^{\prime}$ implies $u, v \in S_{\beta}$ for some $\beta \geq \alpha$, since otherwise $\phi_{\alpha}$ is not defined. We have $L_{\alpha}^{\prime}, R_{\alpha} \subseteq\left(A_{\alpha}^{\prime}\right)^{*}$ for all $\alpha \in Y$.

Defining $R_{\alpha}^{\prime}=\left\{w \in\left(A_{\alpha}^{\prime}\right)^{*}: w \in S_{\alpha}\right\}$, we have $R_{\alpha}=R_{\alpha}^{\prime} \# R_{\alpha}^{\prime}$ (since membership of $w$ in $S_{\alpha}$ depends only on the content of $w$ ). The language $R_{\alpha}^{\prime}$ is recognised by a finite automaton consisting of the semilattice $Y$ with an adjoined top element $T$ as the start state, and final state $\alpha$. The transition function is given by the meet operation: $(\top, x) \mapsto \gamma$ and $(\beta, x) \mapsto \beta \wedge \gamma$ for $x \in A_{\gamma}$. A word $w$ is accepted by this automaton if and only if the meet of all $\gamma$ such that $w$ contains a symbol in $A_{\gamma}$ is $\alpha$. Thus $R_{\alpha}^{\prime}$ is regular, and hence so is $R_{\alpha}$, as a concatenation of regular languages.

Now choose a homomorphism $\psi_{\alpha}:\left(A_{\alpha}^{\prime}\right)^{*} \rightarrow A_{\alpha}^{*}$ defined by $x \mapsto w_{x}$ such that $w_{x}=_{S} x \phi_{\alpha}$. Let $W=\left\{w_{x}: w \in A_{\alpha}^{*}\right\}$ and $M=\operatorname{WP}\left(S_{\alpha}, A_{\alpha}\right) \cap W^{*}$. Then $M \in \mathfrak{C}$, and $L_{\alpha}^{\prime}$ is the inverse image of $M$ under the gsm-mapping from $\left(A_{\alpha}^{\prime}\right)^{*} \#\left(A_{\alpha}^{\prime}\right)^{*}$ to $\left(A_{\alpha}\right)^{*} \#\left(A_{\alpha}\right)^{*}$ that preserves \# and maps all symbols in $x$ before the \# to $x \psi_{\alpha}$ and all symbols $x$ after the \# to $\left(x \psi_{\alpha}\right)^{\text {rev. }}$. Since $\mathfrak{C}$ is closed under inverse gsm-mappings, $L_{\alpha}^{\prime}$ is thus in $\mathfrak{C}$. In turn, $L_{\alpha}$ is in $\mathfrak{C}$, hence so is $\operatorname{WP}(S, A)$, as the union of the finitely many $L_{\alpha}$.

The class $\mathcal{D C F}$ is not closed under finite union [HU79, Theorem 10.5(b)]. We conjecture that a finitely generated strong semilattice of $U(\mathcal{D C F})$ semigroups need not be $U(\mathcal{D C F})$. Let $Y=\{\alpha, \beta\}$ be a two-element semilattice with $\alpha>\beta$. Let $S_{\alpha}$ be the free group generated by $\{x, y\}$ and let $S_{\beta}$ be $\mathbb{Z}$ (under + ). Define $\phi_{\alpha, \beta}$ to be the homomorphism extending $x \mapsto 1, y \mapsto-1$. Both $S_{\alpha}$ and $S_{\beta}$ are virtually free groups and so $U(\mathcal{D C F})$, but the word problem of $\mathcal{S}\left[Y ;\left\{S_{\alpha}, S_{\beta}\right\} ; \phi_{\alpha, \beta}\right]$ does not appear to be deterministic context-free, 
for checking equality in $S_{\alpha}$ seems to require computing reduced words on the stack, while checking equality in $\mathbb{Z}$ seems to require using the stack as a counter, and there is no way to know in advance which is required.

\section{REES MATRIX SEMIGROUPS}

Let us recall the definition of a Rees matrix semigroup. Let $S$ be a semigroup, let $I$ and $\Lambda$ be abstract index sets, and let $P \in \operatorname{Mat}_{\Lambda \times I}(S)$ (that is, $P$ is a $\Lambda \times I$ matrix with entries from $S)$. Denote the $(\lambda, i)$-th entry of $P$ by $p_{\lambda i}$. The Rees matrix semigroup over $S$ with sandwich matrix $P$, denoted $\mathcal{M}[S ; I, \Lambda ; P]$, is the set $I \times S \times \Lambda$ with multiplication defined by

$$
(i, x, \lambda)(j, y, \mu)=\left(i, x p_{\lambda j} y, \mu\right) .
$$

This construction is important because it arises in the classification of completely simple semigroups as Rees matrix semigroups over groups; see How95, Sect. 3.2-3.3].

Hoffmann et al. showed that a completely simple semigroup is $U(\mathcal{C F})$ if and only if it is isomorphic to a Rees matrix semigroup over a finitely generated virtually free group [HHOT12, Theorem 2]; their proof depends on virtually free groups having deterministic context-free word problem. The following theorem generalizes Hoffmann et al.'s characterization to Rees matrix semigroups over arbitrary semigroups. See [GG66, HU79] for background on inverse gsm-mappings.

Theorem 8.1. Let $\mathfrak{C}$ be a class of languages closed under inverse gsmmappings and intersection with regular languages (in particular, $\mathcal{C F}$ or $\mathcal{D C F}$ ). Then a finitely generated Rees matrix semigroup over a semigroup $S$ is $U(\mathfrak{C})$ if and only if $S$ is $U(\mathfrak{C})$.

Proof. Let $M=\mathcal{M}[S ; I, \Lambda ; P]$ be a Rees matrix semigroup and let $\mathfrak{C}$ be as in the statement of the theorem. If $M$ is $U(\mathfrak{C})$, then it must be finitely generated, hence $S$ is also finitely generated and thus $U(\mathfrak{C})$.

Conversely, suppose that $S$ is $U(\mathfrak{C})$ and $M$ is finitely generated by $B \subseteq$ $I \times S \times \Lambda$, and let $A$ be the projection of $B$ onto $S$. For each $i \in I$ and $\lambda \in \Lambda$, choose a word $w_{\lambda i} \in A^{*}$ representing $p_{\lambda i}$. Let $W$ be the (finite) set of all the $w_{\lambda i}$. Let $L=\operatorname{WP}(S, A) \cap(A W)^{*} A \# A(W A)^{*}$, which is in $\mathfrak{C}$, as the intersection of a language in $\mathfrak{C}$ with a regular language. We will define a gsm-mapping $\Phi$ such that $\operatorname{WP}(M, B)$ is the inverse image of $L$ under $\Phi$.

First, define a gsm-mapping $\phi: B^{*} \rightarrow A^{*}$ by

$$
\left(i_{1}, x_{1}, \lambda_{1}\right) \ldots\left(i_{m}, x_{m}, \lambda_{m}\right) \mapsto x_{1} w_{\lambda_{1} i_{2}} x_{2} \ldots w_{\lambda_{m-1} i_{m}} x_{m} .
$$

Then for $w=\left(i_{1}, x_{1}, \lambda_{1}\right) \ldots\left(i_{m}, x_{m}, \lambda_{m}\right)$ we have $w={ }_{M}(i(w), w \phi, \lambda(w))$, where $i(w):=i_{1}$ and $\lambda(w):=\lambda_{m}$.

Now extend $\phi$ to a gsm-mapping $\Phi:(B \cup\{\#\})^{*} \rightarrow(A \cup\{\#\})^{*}$ as follows: For $u, v \in B^{*}$ and $w \in(B \cup\{\#\})^{*}$, let $\left(u \# v^{\mathrm{rev}}\right) \Phi=u \phi \#(v \phi)^{\mathrm{rev}} c$, where $c=\varepsilon$ if $i(u)=i(v)$ and $\lambda(u)=\lambda(v)$, and $c=\#$ otherwise. (Since $I$ and $\Lambda$ are finite, the computation of $c$ can be done by storing $i(u)$ and $\lambda(u)$ in the state and then checking against $\lambda(v)$ and $i(v)$.) Let $\left(u \# v^{\mathrm{rev}} \# w\right) \Phi=$ $u \phi \#(v \phi)^{\mathrm{rev}} \#$ (achieved by storing in the state whether \# has already been seen). 
The preimage of $L$ in $(B \cup\{\#\})^{*}$ under $\Phi$ consists of all words of the form $u \# v^{\mathrm{rev}}$ with $u, v \in B^{*}$ such that $i(u)=i(v), \lambda(u)=\lambda(v)$ and $u \phi \#(v \phi)^{\mathrm{rev}} \in$ $\mathrm{WP}(S, A)$. But this is exactly all $u \# v^{\mathrm{rev}}$ such that $u={ }_{M} v$, so $L \Phi^{-1}=$ $\mathrm{WP}(M, B)$. Hence $M$ is $U(\mathfrak{C})$, since its word problem is obtained from a language in $\mathfrak{C}$ by an inverse gsm-mapping.

The fact that every completely regular semigroup is isomorphic to a semilattice (not necessarily strong) of completely simple semigroups How95, Theorem 4.1.3] raises the following question:

Question 8.2. Which completely regular semigroups are $U(\mathcal{C F})$ ?

\section{BRUCK-REILLY EXTENSIONS}

Let $M$ be a monoid with presentation $\langle A \mid \mathcal{R}\rangle$ and $\phi: M \rightarrow M$ an endomorphism. The Bruck-Reilly extension $\operatorname{BR}(M, \phi)$ of $M$ by $\phi$ is the monoid with presentation $\langle A, b, c \mid \mathcal{R}, b c=1, b a=(a \phi) b, a c=c(a \phi) \quad(a \in A)\rangle$. This is an analogue for monoids of the notion of HNN-extensions for groups.

If $\phi$ is the identity endomorphism, then $\operatorname{BR}(M, \phi)$ is isomorphic to the direct product of $M$ with the bicyclic monoid generated by $\{b, c\}$. Thus by Lemma 5.3 the class of $U(\mathcal{C F})$ semigroups is not closed under BruckReilly extensions. In Lemmata 9.1 to 9.4 below, we establish a necessary and sufficient condition for $\operatorname{BR}(M, \phi)$ to be $U(\mathcal{C F})$.

Lemma 9.1. Let $M$ be a monoid and $\phi: M \rightarrow M$ an endomorphism. If $\operatorname{BR}(M, \phi)$ is $U(\mathcal{C F})$, then $M$ is $U(\mathcal{C F})$.

Proof. Let $Z$ be a generating set for $\operatorname{BR}(M, \phi)$, and let $Z^{\prime}=Z \cap M$. Then $M$ is generated by

$$
Y=\left\{z \phi^{n}: z \in Z^{\prime}, n \in \mathbb{N} \cup\{0\}\right\} .
$$

If every $z \in Z^{\prime}$ has a finite orbit under $\phi$, then $Y$ is finite and so $M$ is a finitely-generated submonoid of $\operatorname{BR}(M, \phi)$ and so $U(\mathcal{C F})$. So suppose, with the aim of obtaining a contradiction, that $z \in Z^{\prime}$ has infinite orbit under $\phi$ : that is, that all the elements $z \phi^{n}$ (where $n \in \mathbb{N}$ ) are distinct.

Lemma 9.2. Let $\beta, \beta^{\prime}, \delta, \delta^{\prime} \in \mathbb{N} \cup\{0\}$. Then there exist $\alpha, \alpha^{\prime}, \gamma, \gamma^{\prime} \in \mathbb{N} \cup$ $\{0\}$ such that $c^{\gamma} b^{\beta} z c^{\delta} b^{\alpha}={ }_{\mathrm{BR}(M, \phi)} c^{\gamma^{\prime}} b^{\beta^{\prime}} z c^{\delta^{\prime}} b^{\alpha^{\prime}}$ if and only if $\max \{\beta, \delta\}=$ $\max \left\{\beta^{\prime}, \delta^{\prime}\right\}$.

Proof. The normal form of $c^{\gamma} b^{\beta} z c^{\delta} b^{\alpha}$ is $c^{\gamma-\beta+\max \{\beta, \delta\}} z \phi^{\max \{\beta, \delta\}} b^{\alpha-\delta+\max \{\beta, \delta\}}$; the same holds replacing $\gamma, \beta, \alpha, \delta$ by their dashed versions.

First, suppose that there exist $\alpha, \alpha^{\prime}, \gamma, \gamma^{\prime}$ such that $c^{\gamma} b^{\beta} z c^{\delta} b^{\alpha}={ }_{\mathrm{BR}(M, \phi)}$ $c^{\gamma^{\prime}} b^{\beta^{\prime}} z c^{\delta^{\prime}} b^{\alpha^{\prime}}$. By the previous paragraph, since all $z \phi^{n}$ are distinct, it follows that $\max \{\beta, \delta\}=\max \left\{\beta^{\prime}, \delta^{\prime}\right\}$.

Now suppose that $\max \{\beta, \delta\}=\max \left\{\beta^{\prime}, \delta^{\prime}\right\}$. Choose $\alpha$ and $\gamma$ such that $\gamma=\beta-\max \{\beta, \delta\}$ and $\alpha=\delta-\max \{\beta, \delta\}$, and similarly for dashed versions. Then, by the first paragraph, $c^{\gamma} b^{\beta} z c^{\delta} b^{\alpha}={ }_{\mathrm{BR}(M, \phi)} c^{\gamma^{\prime}} b^{\beta^{\prime}} z c^{\delta^{\prime}} b^{\alpha^{\prime}}$.

Let $L$ be the word problem of $\operatorname{BR}(M, \phi)$ with respect to $Z \cup\{b, c\}$; the language $L$ is context-free. Let

$$
K=L \cap c^{*} b^{*} z c^{*} b^{*} \# b^{*} c^{*} z b^{*} c^{*} .
$$


Then $K$ is context-free. Let $J$ be the language obtained from $K$ by applying the gsm-mapping that deletes any initial sequence of symbols $c$, any terminal sequence of symbols $c$, any sequence of symbols $b$ immediately before \#, and any sequence of symbols $b$ immediately after \#. Thus $J$ consists of words of the form $b^{\beta} z c^{\delta} \# c^{\delta^{\prime}} z b^{\beta^{\prime}}$ for which there exist $\alpha, \alpha^{\prime}, \gamma, \gamma^{\prime} \in \mathbb{N} \cup\{0\}$ such that $c^{\gamma} b^{\beta} z c^{\delta} b^{\alpha} \# b^{\alpha^{\prime}} c^{\delta^{\prime}} z b^{\beta^{\prime}} c^{\gamma^{\prime}} \in K$. Since $K$ is a subset of the word problem for $\operatorname{BR}(M, \phi)$, it follows from the lemma that $J$ is the language

$$
\left\{b^{\beta} z c^{\delta} \# c^{\delta^{\prime}} z b^{\beta^{\prime}}: \max \{\beta, \delta\}=\max \left\{\beta^{\prime}, \delta^{\prime}\right\}\right\} .
$$

A straightforward argument using the pumping lemma shows that $J$ cannot be context-free. However, the class of context-free languages is closed under gsm-mappings, and $J$ was obtained from the context-free language $K$ via a gsm-mapping. This is a contradiction, which completes the proof.

Lemma 9.3. If $\operatorname{BR}(M, \phi)$ is $U(\mathcal{C F})$, then $\operatorname{im} \phi^{n}$ is finite for some $n$.

Proof. Suppose $S=\operatorname{BR}(M, \phi)$ is $U(\mathcal{C F})$. As a corollary of the preceding result, $M$ admits a finite generating set $X$, and so $S$ is generated by $Y=$ $X \cup\{b, c\}$. Let $L$ be the word problem of $S$ with respect to $Y$. Let $K=$ $X^{*} b^{*} \# X^{*} b^{*} \cap L$; then $K$ is also context-free. Let $n$ be greater than the pumping constant for $K$.

Let $u \in X^{*} \cap \operatorname{im} \phi^{n}$. Then there exists $v \in X^{*}$ such that $v \phi^{n}=u$. Therefore $b^{n} v=_{S}\left(v \phi^{n}\right) b^{n}={ }_{S} u b^{n}$. Therefore $u b^{n} \# v^{\text {rev }} b^{n} \in K$.

Suppose that $|v|>n$. Applying the pumping lemma shows that $u b^{n} \# v^{\text {rev }} b^{n}$ factors as pqrst with $|r|<n$ such that $p q^{i} r s^{i} t$ also lies in $K$ for any $i$. By the definition of $K$, the factors $q$ and $s$ must lie wholly within the words $u$, $b^{n}, v^{\mathrm{rev}}$, or $b^{n}$. By the bound on its length, the factor $r$ cannot involve the whole of the left-hand $b^{n}$ or the whole of $v^{\text {rev }}$. Further, it is impossible that $q$ and $s$ are both non-empty and lie in $b^{n}$ and $v^{\text {rev }}$, respectively, for taking $i=2$ gives a word with different numbers of symbols $b$ before and after the \#. Thus either $q$ lies in $u$ and $s$ is empty, or else $q$ is empty and $s$ lies in $v^{\mathrm{rev}}$.

In the former case, taking $i=0$ yields a word in $K$ of the form $\hat{u} b^{n} \# v^{\text {rev }} b^{n}$, where $|\hat{u}|<|u|$. Thus $\hat{u} b^{n}={ }_{S} b^{n} v={ }_{S}\left(v \phi^{n}\right) b^{n}$, and so $\hat{u}=v \phi^{n}=u$. Thus we can replace $u$ by a strictly shorter word $\hat{u}$ that is equal to it in $S$ (and so in $M$ ) and repeat the above reasoning.

In the latter case, taking $i=0$ yields a word in $K$ of the form $u b^{n} \# \hat{v}^{\text {rev }} b^{n}$, where $|\hat{v}|<|v|$. Thus $u b^{n}={ }_{S} b^{n} \hat{v}=\left(\hat{v} \phi^{n}\right) b^{n}$. Thus we can replace $v$ by a strictly shorter word $\hat{v}$ that has same image under $\phi^{n}$.

These replacements do not alter the element $u=v \phi^{n}$ of $\operatorname{im} \phi^{n}$. However, since these replacements are always by strictly shorter words, this replacement process, which began with the assumption that $|v|>n$, must reach a state where $|v| \leq n$. That is, $\operatorname{im} \phi^{n} \subseteq X^{\leq n} \phi^{n}$ and is thus finite.

Lemma 9.4. Let $M$ be a $U(\mathcal{C F})$ monoid and $\phi: M \rightarrow M$ an endomorphism such that $\mathrm{im} \phi^{n}$ is finite for some $n$. Then the Bruck-Reilly extension $\operatorname{BR}(M, \phi)$ is $U(\mathcal{C F})$.

Proof. Let $S=\operatorname{BR}(M, \phi)$. Let $T=\operatorname{im} \phi^{n}$, and let $i$ be the index and $p$ the period of the action of $\phi$ on the finite monoid $T$. Let $m=n+i+p-1$, 
so we have $\phi^{m+1}=\phi^{n+i}$. For $\beta \in \mathbb{N}_{0}$, let $[\beta]$ denote the unique element of $\{0, \ldots, m\}$ such that $\phi^{\beta}=\phi^{[\beta]}$.

Let $X$ be a finite generating set for $M$ with $T \subseteq X$, and let $\mathcal{B}$ be a pushdown automaton recognising $\operatorname{WP}(M, X)$, with states $Q$, initial state $q_{0}$ and stack alphabet $\Gamma$, with bottom marker $\perp \in \Gamma$, which is never pushed or popped during computations in $\mathcal{B}$. For $z \in X^{*}$, we will denote the stack contents (including $\perp$ ) of $\mathcal{B}$ after reading $z$ from the start state $q_{0}$ by $s_{z}$, and the state reached at that point by $q_{z}$. Of course if $\mathcal{B}$ is non-deterministic then $s_{z}$ and $q_{z}$ are not necessarily unique. We use the notation to represent any possible choice.

Let $Y=X \cup\{b, c\}$. We will define a pushdown automaton $\mathcal{A}$ recognising $\mathrm{WP}(S, Y)$. The idea behind the automaton is that while reading a word $u \in Y^{*}$, it can use its stack both to record the $c^{\alpha-} b^{\beta}$ part of the the BruckReilly normal form, while also simulating $\mathcal{B}$ : the stack contents will be $c^{\alpha}$ [simulated stack of $\left.\mathcal{B}\right] b^{\beta}$. The automaton $\mathcal{A}$ tracks of the number of times $\phi$ must be applied to the next subword from $X^{*}$ as a result of uncancelled symbols $b$ already read, and guesses (and records and later checks) how many further applications of $\phi$ are required due to future uncancelled symbols $c$. This guess need only be one of a finite number of possibilities, since there are only $m$ distinct actions of powers of $\phi$ on $M$. The key to this working is that whenever the automaton needs to access stack symbols from $\Gamma$, there will be a bounded amount of information above these symbols and so they can be accessed without destroying the information above. If $u={ }_{S} c^{\alpha} \hat{u} b^{\beta}$, the information the automaton ends up storing about $\hat{u}$ is comprised of two components: a word $s_{z}$ in $\Gamma^{*}$ on the stack, and a symbol $t$ from $T$ in the state, such that $\hat{u}=z t$. Upon then reading $\# v^{\text {rev }}$ for $v \in Y^{*}$, similar reasoning allows us to check $v^{\text {rev }}$ against the stored 'normal form' to determine whether $u={ }_{S} v$.

The states of $\mathcal{A}$ are divided into 'pre-\#' and 'post-\#' states, and we begin by describing the behaviour in the pre-\# states. These states are of the form $(q, t, k,-)$ or $(q, t, k, l)$, where $q \in Q, t \in T^{1}, 0 \leq k \leq m$ and $l \in\{0, \ldots, \max \{0, n-k\}\}$. The start state of $\mathcal{A}$ is $\left(q_{0}, 1,0,-\right)$, where 1 is the adjoined identity in $T^{1}$ (not equal to the identity element of $T$ ).

Our goal is that after reading a word $w \in Y^{*}$, for some word $z \in X^{*}$ the automaton is in the following configuration:

- If $w=c^{\alpha}$, the stack contents are $C^{\alpha}$. Otherwise, the stack contents are of the form $C^{\alpha} s_{z} B_{\beta}$ for $0 \leq \beta \leq n+i$ or $C^{\alpha} s_{z} B_{n+i} B^{\beta-n-i}$ for $\beta>n+i$.

- If $w \in\{b, c\}^{*}$, then the state is $\left(q_{0}, 1,[\beta],-\right)$. Otherwise, the state is $\left(q_{z}, t,[\beta], l\right)$, where $l$ represents a guess regarding the number of symbols $c$ still to be read. If $l$ takes the maximum value $n-[\beta]$, the guess is that there are still at least that many symbols $c$ to be read, whereas for other values of $l$ the guess is exact.

- If the last state component is blank, then $z=\varepsilon$ and $w={ }_{S} c^{\alpha} b^{\beta}$. If $l=0$, then $w={ }_{S} c^{\alpha} z t b^{\beta}$. Otherwise $w c^{\beta+l}={ }_{S} c^{\alpha} z t$.

The state component $k$ records the number of times $\phi$ needs to be applied to the next upcoming subword from $X^{*}$ as a result of symbols $b$ already read. The state component $l$ represents a guess at the number of times $\phi$ 
needs to be applied to the same subword as a result of uncancelled symbols $c$ still to be read. If $\phi$ is to be applied at least $n$ times, then the element represented falls into $T$ and we begin recording it in the state instead, in the component $t$. The 'guess' at the value of $l$ occurs when the automaton first reads a symbol from $X$, and the idea is that we will be able to tell when we have read the right number of symbols $c$ by the final state component being 0 . The automaton has the property that if our initial guess is the maximum possible, then $t \neq 1$ once the guess has been made. This allows us to detect when a non-maximal guess is incorrect: the automaton fails if a $c$ is read from a state of the form $(q, 1, k, 0)$, as this will imply we have seen more symbols $c$ than we had guessed.

We now describe the behaviour of $\mathcal{A}$ in a state $(q, t, k,-)$ or $(q, t, k, l)$ on input from $Y$. Observe that the stack contents are always in $C^{*} \Gamma^{*} S_{B}$ or in $C^{*} \Gamma^{*} B_{n+i} B^{*}$, where $S_{B}=\left\{B_{1}, \ldots, B_{n+i-1}\right\}$. Note that some transitions technically require more than one state to execute, but we do not name the intermediate states involved in these transitions, the important point being that there are finitely many of them in total. Choose a map $\psi: X \rightarrow X^{*}$ such that $x \psi={ }_{M} x \phi$ for all $x \in X$.

- On input $b$ : if the stack is empty or the top-of-stack is $C$, push $\perp B_{1}$; if the top-of-stack is in $X$, push $B_{1}$; if the top-of-stack is $B_{j}$ for $j<n+i$, replace it by $B_{j+1}$; if the top-of-stack is $B_{n+i}$ or $B$, push $B$. In all cases, replace $k$ by $[k+1]$. This ensures that after reading $b^{\beta}$ from the start state, the third component of the state is $[\beta]$.

- On input $c$ : if $B$ at top of stack, pop $B$ and replace $k$ by $k-1$ unless $k=n+i$ and the next symbol on the stack is also $B$, in which case replace $k$ by $m$; if $C$ is at the top of the stack, add another $C$ (this can only occur while $t=1$ ); if $x \in X$ is at the top of the stack, replace $l$ by $l-1$ unless $l=0$, in which case we fail if $t=1$, and replace $t$ by $t \phi$.

- On input $x \in X$, if the last state component is blank, we first replace it by either 0 if $k \geq n$, or otherwise some non-deterministically chosen $l \in\{0, \ldots, n-k\}$, before proceeding. When the last state component is not blank: if $k+l \geq n$, replace $t$ by $t\left(x \phi^{[k+l]}\right) \in T$, without altering the stack; otherwise, the top-of-stack will be $B_{k}$, and we pop this from the stack, then simulate reading $x \psi^{k+l}$ from state $q$ in the automaton $\mathcal{B}$, replacing $q$ by the state reached and finally adding $B_{k}$ to the top of the stack.

Next, we describe the 'transition' phase of $\mathcal{A}$, when \# is read from a pre-\# state $(q, t, k,-)$ or $(q, t, k, l)$.

- If the last state component is blank, this means that we read no symbols from $X$. The state will be $\left(q_{0}, 1,[\beta],-\right)$ and the stack contents $c^{\alpha} b^{\beta}$. Move to state $\left(q_{\#}, 1,1,[\beta], 0\right)_{\#}$. (Recall that $q_{\#}$ denotes a state reached after reading $\#$ in $\mathcal{B}$ from state $q_{0}$.) If moreover the top-of-stack is $C$, then we had $u=c^{\alpha}$ and so the stack contents are $C^{\alpha}$. In this case we move to a special state $f$, without altering the stack. 
- If $l \neq 0$, the automaton fails, since this means we have not read as many symbols $c$ as we guessed, so that $\phi$ has not been applied the correct number of times.

- If $l=0$, move to state $(q, t, 1,[\beta], 0)_{\#}$.

Note that after the transition phase, $\mathcal{A}$ is in a 'post-\#' state of the form $(q, t, 1, k, l)_{\#}$, or in the state $f$. In general the post-\# states other than $f$ have the form $\left(q, t, t^{\prime}, k, l\right)_{\#}$ or $\left(q, t, t^{\prime}, k, l\right)$ for $q \in Q, t, t^{\prime} \in T^{1}, 0 \leq l \leq m$ and $k \in\{0, \ldots, \max \{0, n-l\}\}$. The subscript \# indicates that the symbol \# has not yet been simulated in $\mathcal{B}$. We first describe the action of $b$ and $c$ in these states. A new set of stack symbols $S_{D}=\left\{D_{1}, \ldots, D_{n+i}, D\right\}$ is introduced at this stage to count uncancelled occurrences of $c$. Note that in these states the bottom-of-stack marker $\perp$ from $\mathcal{B}$ is always still on the stack, so it is not possible for the top-of-stack to be $C$.

- On input $c$ : If top-of-stack is $B, B_{j}$ or $\gamma \in \Gamma$, push $D_{1}$ onto stack; if top-of-stack is $D_{j}$ for $j<n+i$, replace it by $D_{j+1}$; if top-of-stack is $D_{n+i}$ or $D$, push $D$ onto stack. In all cases, replace $l$ by $[l+1]$.

- On input $b$ : If top-of-stack is $B$ or $B_{1}$, pop it; if top-of-stack is $B_{j}$ for $j>1$, replace it by $B_{j-1}$; in these two cases replace $k$ by $k-1$, unless $k=n+i$ and the top two stack symbols are both $B$, in which case replace $k$ by $m$. If top-of-stack is $D$ or $D_{1}$, pop it; if top-of-stack is $D_{j}$ for $j>1$, replace it by $D_{j-1}$; in these two cases replace $l$ by $l-1$, or by $m$ if $l=n+i$ and the top two symbols are both $D$. The transitions for $B$ and $B_{j}$ cancel final symbols $b$ from the normal form of $v^{\text {rev }}$ against those from $u$. The transitions for $D$ and $D_{j}$ apply the relation $b c=1$ in reverse and ensure that if $v=c^{\gamma} z b^{\delta}$ then the final state component is $[\gamma]$ after reading $v^{\text {rev }}$. If top-of-stack is $\gamma \in \Gamma$, the automaton fails, since this means that the normal form of $v$ contains more symbols $b$ than the normal form of $u$.

Suppose that after reading $u \in Y^{*}$, the automaton was in state $(q, t, k, 0)$ with stack contents $C^{\alpha} s_{z} B^{\beta}$. Then after further reading $\# w^{\text {rev }}$ with $w \in$ $\{b, c\}^{*}$ and $w={ }_{S} c^{\gamma} b^{\delta}$, if $\delta>\beta$ the automaton has failed, and if $\beta \geq \delta$ the stack contents are $C^{\alpha} s_{z} B^{\beta-\delta} D^{\gamma}$ and the state is $(q, t, 1,[\beta-\delta],[\gamma])_{\#}$, unless we have meanwhile non-deterministically transitioned to the state $f$ as described below.

Next, we describe the action of $x \in X$ in a post-\# state $\left(q, t, t^{\prime}, k, l\right)_{\#}$ or $\left(q, t, t^{\prime}, k, l\right)$.

- If $k+l \geq n$, replace $t^{\prime}$ by $\left(x \phi^{[k+l]}\right) t^{\prime} \in T$.

- If $k+l<n$, the top two symbols of the stack are $B_{\delta} D_{\gamma}$. Pop these two symbols, then simulate reading $t(\#) t^{\prime}\left(x \psi^{[k+l]}\right)$ from state $q$ in $\mathcal{B}$ (where (\#) indicates that the \# is present if and only if it was in the subscript of the state), replacing $q$ by the state reached, and then return the two popped symbols to the top of the stack (in the same order). If the state had a \# subscript, remove it to record that \# has now been simulated in $\mathcal{B}$.

Since $\mathcal{B}$ cannot be assumed in general to accept by empty stack, $\mathcal{A}$ has to non-deterministically guess when it has finished receiving input from $X$ : 
- In each state $\left(q, t, t^{\prime}, 0, l\right)_{(\#)}$, there is an $\epsilon$-transition which simulates reading $t(\#) t^{\prime}$ in $\mathcal{B}$. Let $q^{\prime} \in Q$ be the state reached following this computation. Then if $q^{\prime}$ is a final state of $\mathcal{B}$, the automaton deletes all remaining symbols from $\Gamma$ (including $\perp$ ) from the stack and moves to state $f$. Otherwise the automaton fails.

On reaching the state $f$, the stack contents are always of the form $C^{\alpha}$. The state $f$ only accepts input $b$ and $c$, and behaves like the 'post-\#' state of a pushdown automaton for the word problem of the bicyclic monoid:

- On input $c$ : with $C$ at top-of-stack, non-deterministically either pop $C$ or push $D$ (to indicate a guess that there are still symbols $b$ to be read); with $D$ at top-of-stack, or on empty stack, push $D$.

- On input $b$ : fail if top-of-stack is $C$; on top-of-stack $D$, pop $D$.

Final acceptance in $\mathcal{A}$ is by empty stack in state $f$.

Lemmata 9.1, 9.3 and 9.4 combine to give a complete characterisation of the $U(\mathcal{C F})$ monoids occurring as Bruck-Reilly extensions:

Theorem 9.5. Let $M$ be a monoid and $\phi: M \rightarrow M$ an endomorphism. Then $\operatorname{BR}(M, \phi)$ is $U(\mathcal{C F})$ if and only if $M$ is $U(\mathcal{C F})$ and $\operatorname{im} \phi^{n}$ is finite for some $n$.

The bicyclic monoid arises as the submonoid generated by $\{b, c\}$ in any Bruck-Reilly extension. As discussed in Section 3, we conjecture that this monoid is not $U(\mathcal{D C F})$, and hence no Bruck-Reilly extension is $U(\mathcal{D C F})$.

\section{Further open PROBlems}

Question 10.1. Does every cancellative $U(\mathcal{C F})$ semigroup have deterministic context-free word problem?

Question 10.2. Is it possible to characterize the commutative (respectively, cancellative, inverse) $U(\mathcal{C F})$ semigroups?

The previous two questions are motivated by the group case, since the classes of $U(\mathcal{C F})$ and $U(\mathcal{D C F})$ groups coincide and are precisely the virtually free groups. In particular, the abelian $U(\mathcal{C F})$ groups are thus either finite or of the form $\mathbb{Z} \times F$, where $F$ is finite and abelian.

Question 10.3. Does there exist an infinite periodic $U(\mathcal{C F})$ semigroup?

Question 10.4. Must the group of units of a $U(\mathcal{C F})$ monoid be finitely generated?

\section{REFERENCES}

[BC18] T. Brough \& A. J. Cain. 'A language hierarchy of binary relations'. 2018. arXiv: 1805.03125]

[BO93] R. V. Book \& F. Otto. String Rewriting Systems. Texts and Monographs in Computer Science. Springer, 1993.

[Bro10] T. R. Brough. Groups with Poly-Context-Free Word Problem. Ph.d. thesis, University of Warwick, 2010.

[Bro14] T. Brough. 'Groups with poly-context-free word problem'. Groups Complexity Cryptology, 6, no. 1 (2014), pp. 9-29. DOI: $10.1515 /$ gcc-2014-0002 
[Bro18] T. Brough. 'Word problem languages for free inverse monoids'. In S. Konstantinidis \& G. Pighizzini, eds, Descriptional Complexity of Formal Systems, no. 10952 in Lecture Notes in Computer Science, pp. 24-36. Springer, 2018. DOI: 10.1007/978-3-319-94631-3_3

[CM12] A. J. Cain \& V. Maltcev. 'Context-free rewriting systems and word-hyperbolic structures with uniqueness'. International Journal of Algebra and Computation, 22, no. 7 (2012). DOI: 10.1142/S0218196712500610.

[CRRT01] C. M. Campbell, E. F. Robertson, N. Ruškuc, \& R. M. Thomas. 'Automatic semigroups'. Theoretical Computer Science, 250, no. 1-2 (2001), pp. 365-391. DOI: 10.1016/S0304-3975(99)00151-6.

[DG99] A. Duncan \& R. H. Gilman. 'Word hyperbolic semigroups'. Mathematical Proceedings of the Cambridge Philosophical Society, 136, no. 3 (1999), pp. 513-524. DOI: 10.1017/S0305004103007497.

[Dun85] M. J. Dunwoody. 'The accessibility of finitely presented groups'. Inventiones Mathematicae, 81, no. 3 (1985), pp. 449-457. DOI: 10.1007/BF01388581

$\left[\mathrm{ECH}^{+} 92\right]$ D. B. A. Epstein, J. W. Cannon, D. F. Holt, S. V. F. Levy, M. S. Paterson, \& W. P. Thurston. Word Processing in Groups. Jones \& Bartlett, Boston, MA, 1992.

[GG66] S. Ginsburg \& S. Greibach. 'Deterministic context free languages'. Information and Control, 9, no. 6 (1966), pp. 620-648. DOI: 10.1016/S0019-9958(66)80019-0

[Gil02] R. H. Gilman. 'On the definition of word hyperbolic groups'. Mathematische Zeitschrift, 242, no. 3 (2002), pp. 529-541. DOI: 10.1007/s002090100356

[Gro87] M. Gromov. 'Hyperbolic Groups'. In S. M. Gersten, ed., Essays in Group Theory, no. 8 in Mathematical Sciences Research Institute Publications, pp. 75-263. Springer-Verlag, 1987.

[HHOT12] M. Hoffmann, D. F. Holt, M. D. Owens, \& R. M. Thomas. 'Semigroups with a context-free word problem'. In Developments in Language Theory, pp. 97-108. Springer, 2012. DOI: 10.1007/978-3-642-31653-1_10

[HKOT02] M. Hoffmann, D. Kuske, F. Otto, \& R. M. Thomas. 'Some relatives of automatic and hyperbolic groups'. In G. M. S. Gomes, J.-É. Pin, \& P. V. Silva, eds, Semigroups, algorithms, automata and languages, p. 379-406, River Edge, NJ, 2002. World Scientific. DOI: 10.1142/9789812776884_0016.

[HOT08] D. F. Holt, M. D. Owens, \& R. M. Thomas. 'Groups and semigroups with a one-counter word problem'. Journal of the Australian Mathematical Society, 85, no. 02 (2008), p. 197. DOI: 10.1017/S1446788708000864.

[How95] J. M. Howie. Fundamentals of Semigroup Theory. No. 12 in London Mathematical Society Monographs: New Series. Clarendon Press, Oxford University Press, New York, 1995.

[HR06] D. F. Holt \& C. E. Röver. 'Groups with indexed co-word problem'. International Journal of Algebra and Computation, 16, no. 5 (2006), pp. 985-1014. DOI: 10.1142/S0218196706003359.

[HRRT99] D. F. Holt, S. Rees, C. E. Röver, \& R. M. Thomas. 'Groups with contextfree co-word problem'. Journal of the London Mathematical Society, 71, no. 3 (1999), pp. 643-657. DOI: 10.1112/S002461070500654X.

[HT93] T. Herbst \& R. M. Thomas. 'Group presentations, formal languages and characterizations of one-counter groups'. Theoretical Computer Science, 112, no. 2 (1993), pp. 187-213. DOI: 10.1016/0304-3975(93)90018-O

[HU79] J. E. Hopcroft \& J. D. Ullman. Introduction to Automata Theory, Languages, and Computation. Addison-Wesley, Reading, MA, 1st edition, 1979.

[MS83] D. E. Muller \& P. E. Schupp. 'Groups, the theory of ends, and context-free languages'. Journal of Computer and System Sciences, 26, no. 3 (1983), pp. 295-310. DOI: 10.1016/0022-0000(83)90003-X

[RRW98] E. F. Robertson, N. Ruškuc, \& J. Wiegold. 'Generators and relations of direct products of semigroups'. Transactions of the American Mathematical Society, 350, no. 07 (1998), pp. 2665-2686. DOI: 10.1090/S0002-9947-98-02074-1 
Centro de Matemática e Aplicações, Faculdade de Ciências e Tecnologia, Universidade Nova de Lisboa, 2829-516 Caparica, Portugal

E-mail address: tarabrough@gmail.com

Centro de Matemática e Aplicações, Faculdade de Ciências e Tecnologia, Universidade Nova de Lisboa, 2829-516 Caparica, Portugal

E-mail address: a.cain@fct.unl.pt

School of Computer Science, University of St Andrews, North Haugh, St Andrews, Fife KY16 9SX, United Kingdom

E-mail address: markus.pfeiffer@st-andrews.ac.uk 